\title{
The prediction and assessment of the intrauterine fetal with umbilical cord around neck well-being by daily fetal movement counting chart (DFMC)
}

zijun li ( $\square$ lizijunclark@126.com )

Department of Obstetric of Zhejiang Quhua Hospital

\section{Xin Zhou}

Department of Obstetric of the first affiliated hospital of Nanjing Medical University

\section{Yaqin Zheng}

the Clinical laboratory centre of Zhejiang Quhua Hospital

Min Liu

Department of Obstetric of Zhejiang Quhua Hospital

\section{Xiaoying Fu}

Department of Obstetric of Zhejiang Quhua Hospital

Aimei Ye

Department of Obstetric of Zhejiang Quhua Hospital

\section{Research Article}

Keywords: DFMC (daily fetal movement counting chart), FM (fetal movement), Umbilical cord around neck (UCAN), intrauterine fetal safety

Posted Date: February 19th, 2021

DOl: https://doi.org/10.21203/rs.3.rs-197819/v1

License: (c) (1) This work is licensed under a Creative Commons Attribution 4.0 International License. Read Full License 


\section{Abstract \\ Background}

Currently, FM is a sole subjective index which depends on self-assessment of pregnant women, and it can objectively reflect the well being of intrauterine fetus. It has been the focus of obstetricians' research and attention how to quantify fetal movement (FM) and change it into a relatively objective evaluation index. We sought to predict and evaluate the well being of intrauterine fetal with umbilical cord around the neck (UCAN) by performance of daily fetal movement counting chart (DFMC).

\section{Methods}

To retrospectively select 100 -case pregnant women who met the enrolled requirements, and whether the fetal was intrauterine safety or not was predicted by analysis of the DFMC before delivery. According to the different perinatal outcomes after delivery, 100-cases pregnant women were divided into intrauterine safety (IUS) group (44 cases) and intrauterine unsafe (IUNS) group (56 cases). And the independent risk factors of fetal intrauterine safety, including maternal age, gestational week, umbilical artery systolic blood flow to diastolic blood flow ratio (S/D), amniotic fluid index (AFI), modified Fischer score of non stress test (NST), DFMC and mode of delivery were analyzed by multi-factors Binary Logistic Regression.

\section{Results}

Only FM and mode of delivery were independent risk factors affecting fetal safety intrauterine (all $p$ value $<0.05$ ). There was no remarkable difference between DFMC prediction and clinical retrospective evaluation of fetal safety in natural delivery and forceps delivery (all $P$ value $>0.05$ ), except in cesarean section $(p<0.05)$. The sensitivity, specificity and Yuden index of DFMC for predicting the intrauterine safety in natural vaginal delivery were $86.5 \%, 90.0 \%$ and 0.77 respectively, and in cesarean section were $66.7 \%, 94.6 \%$ and 0.61 , respectively. Abnormal DFMC may account for the highest proportion of emergency cesarean section $(87.5 \%, 35 / 40)$.

\section{Conclusions}

DFMC can effectively predict and evaluate the well being of intrauterine fetus with UCAN. At the same time, it can also provide limited clinical proofs for pregnant women with UCAN to choose model of delivery.

\section{Background}

Fetal movement (FM) is a unique behavior mode of mother-to-child communication that reflects the active and passive activities of the fetus in the uterus. FM counting is a long-standing method of 
assessing fetal well being [1]. More than $99 \%$ of women who have given birth to a healthy baby say that it is important to feel the baby's movements every day [2]. When the FM is reduced, they are worried and visit their doctor or health care provider for further evaluations [3]. In women with decreased FM, there is the risk of complications such as fetal growth restriction and stillbirth, especially for those women with pregnancy complications [3,4]. Although FM counting is simple, its usage is controversial. Gradual reduction of $\mathrm{FM}$ and its perception by mother is an important sign of fetal damage that can demonstrate complication $[5,6]$. Maternal perception of reduced FM is the most important marker of decreased fetal activity $[6,7]$. If the mothers carefully count the FM and report on time decrease in fetal movements to physician or health care providers, it is likely to prevent perinatal morbidity and mortality $[3,8]$. In other words, although FM is an objective index of assessment of fetal well-being, it is influenced and interfered by many subjective factors $[3,8,9]$, for example, maternal mood, daily life pattern, behavior change, cognitive ability and individuation difference, etc. Therefore, how to design a strategy for objective, effective and individualized fetal dynamic counting method is the focus of many researchers. Daily FM counting chart (DFMC) is an effective and objective FM counting and recording method, and the DFMC may increase the mother's ability to recognize on time the warning signs and if the fetus is in danger, it will be properly intervened [3, 4]. In this study, we quantified the FM counting and recording method into a relatively objective evaluation index named DFMC, and we could predicate and evaluate the well being of intrauterine fetal with umbilical cord around the neck (UCAN) by performance of DFMC.

\section{Materials And Methods}

\section{Study participants}

This study was conducted with a prospective cohort design and enrolled participants from the department of obstetrics of Zhejiang Quhua Hospital between 2016 Jan and 2020 Jan, and the detailed filtering process is shown in Figure 1. Finally, all selected 100-case pregnant women should meet the following requirements: the gestational age between 37 and 41 weeks, singleton nulliparous women, no high-risk factors confirmed in the assessment before delivery after admission according to the work standard for pregnant women's pregnancy risk assessment and management of Zhejiang province in China [10], UCAN confirmed by B ultrasound before delivery and clinically after delivery, all pregnant women required for participating in the training and teaching of standardized DFMC and passing the test in the outpatient midwifery counseling agency, complete dates including DFMC from 32 weeks of pregnancy to before delivery, and all enrolled patients agreement with acceptance of the suggestion of vaginal delivery.

\section{DFMC and evaluation method}

DFMC based on a quantified FM counting method called "count-to-ten" is a maternal self-counting FM and detailed recording of FM chart data $[11,12]$. To count 3 times at the better active part of the whole day, and the result is recorded in the time period between 09:00 am and 09:00 pm when FM appears the most on the whole day. And 3-time total FM count $\geq 30$ times is defined as normal FM, moreover, while 
the FM after 09:00 pm should be recorded in detail when every time FM count $<10$ times. The term of abnormal FM is strictly defined according to previous research and literature reports $[13,14,15]$, including both FM count and regularity [16]. Three-time consecutive FM count $<10$ times are defined as FM reduction, and irregular FM including excessive FM (e.g. 3-time total FM count $\geq 60$ times) and peak FM in phase of non-peak time FM (e.g. significant changes of the regularity of FM). And absence of FM shows FM disappearance during the periods of peak FM $[13,16]$. The evaluation criteria of DFMC prediction results are defined as follows: the regularity of FM is defined as fetal intrauterine safety, and the reduction, irregular FM and absence of FM are defined as fetal intrauterine insecurity. The detailed recording method described in Figure 2 had showed the FM history of a pregnant woman at 37 weeks of gestation in detail. From 33 to $37^{+0}$ weeks of gestation, FM showed good regularity, not only reflected in the FM peak time (10:30 to 12:30 am everyday) distribution of DFMC, but also normal count distribution (10 beats per half hour) and fluctuation width ( 2 hour/day). In case of accident caused by umbilical cord factors, firstly, the regularity of $\mathrm{FM}$ in $37^{+1}$ week of gestation had changed, the time distribution of the most frequent FMs in a whole day showed a great change from the previous regular peak period of FM to irregular multiple periods of time, the number of FM also increased significantly. Secondly, the FM in $37^{+2}$ week of gestation consciously disappeared completely and this condition lasted near to ten hours from 09:00 am to 70:30 pm. Finally, the fetal heart rate (FHR) was conformed complete disappearance by Doppler ultrasound stethoscope and B-mode ultrasound after admission.

\section{Statistical analysis}

Statistical analysis was performed with SPSS version 19.0 software for Windows (SPSS Inc, Chicago, IL, USA). Descriptive statistics were given as mean, standard deviation, frequency and percentage. The homogeneity test of Levene variance was performed on, independent sample $T$ test was used for comparison between groups. Pearson Chi-square test or Fisher Exact test was used to compare the count data which did not conform to the normal distribution. The risk factors of fetal intrauterine safety were set as a dependent variable and classified into two categories (0: safety, 1: non safety). And age, gestational week, AFI, S/D, UCAN, DFMC, NST and mode of delivery were all set as independent variables, to analyze the high risk factors of fetal intrauterine safety by multi-variables binary logistic stepwise regression analysis, $P<0.05$ was the inclusion criteria. All statistical significance was defined as $P<0.05$.

\section{Date collection}

The maternal age, gestational age, umbilical artery systolic blood flow to diastolic blood flow ratio (S/D), amniotic fluid index (AFI), modified Fischer score of non stress test (NST) and the DFMCs between gestation age of 37 week and 24 hours before delivery were all collected, and perinatal outcomes after delivery were also included, such as 1-minute Apgar score, amniotic fluid cleanliness, the loops of UCAN, neonatal asphyxia or not and mode of delivery, including natural vaginal delivery, cesarean delivery and forceps delivery.

\section{Grouping according to the perinatal outcomes after delivery}


The Apgar score $\leq 6$ at 1 minute, amniotic fluid turbidity $\geq$ II, fetal death in uterus (only 1 case) and neonatal asphyxia were considered as bad perinatal outcomes. One of the four cases mentioned above is defined as fetal intrauterine insecurity, and grouped into intrauterine non safety (IUNS) group ( $n=56)$, and 44-case patients were grouped into intrauterine safety (IUS) group.

\section{Results}

\section{Mode of delivery}

All enrolled 100-case pregnant women accepted the suggestion of vaginal delivery after admission, including one case fetal death in uterus also accepted vaginal delivery by induced labor. This dead fetal was diagnosed as having the UCAN for 4 loops and knotting confirmed by examination after delivery. Thirty-five cases of emergency cesarean section for various reasons after parturition. Selective cesarean section was performed in only five-case pregnant women with a strong desire for cesarean section after parturition. And 47-case pregnant women had experienced successfully natural vaginal delivery, 13 cases had done forceps delivery because of abnormal fetal position.

\section{Prenatal factors of 100-case pregnant women}

The comparison of age, gestational week and AFI between two groups all showed no great significance, all $P$ value $>0.05$. And the comparison of S/D, UCAN, DFMC, NST and mode of delivery all showed remarkable difference between two groups, all $P$ value $<0.05$ (Table 1).

Table 1 Comparison of different risk factors affecting fetal intrauterine safety between different groups $(\mathrm{M} \pm \mathrm{SD})$, [n (\%)].

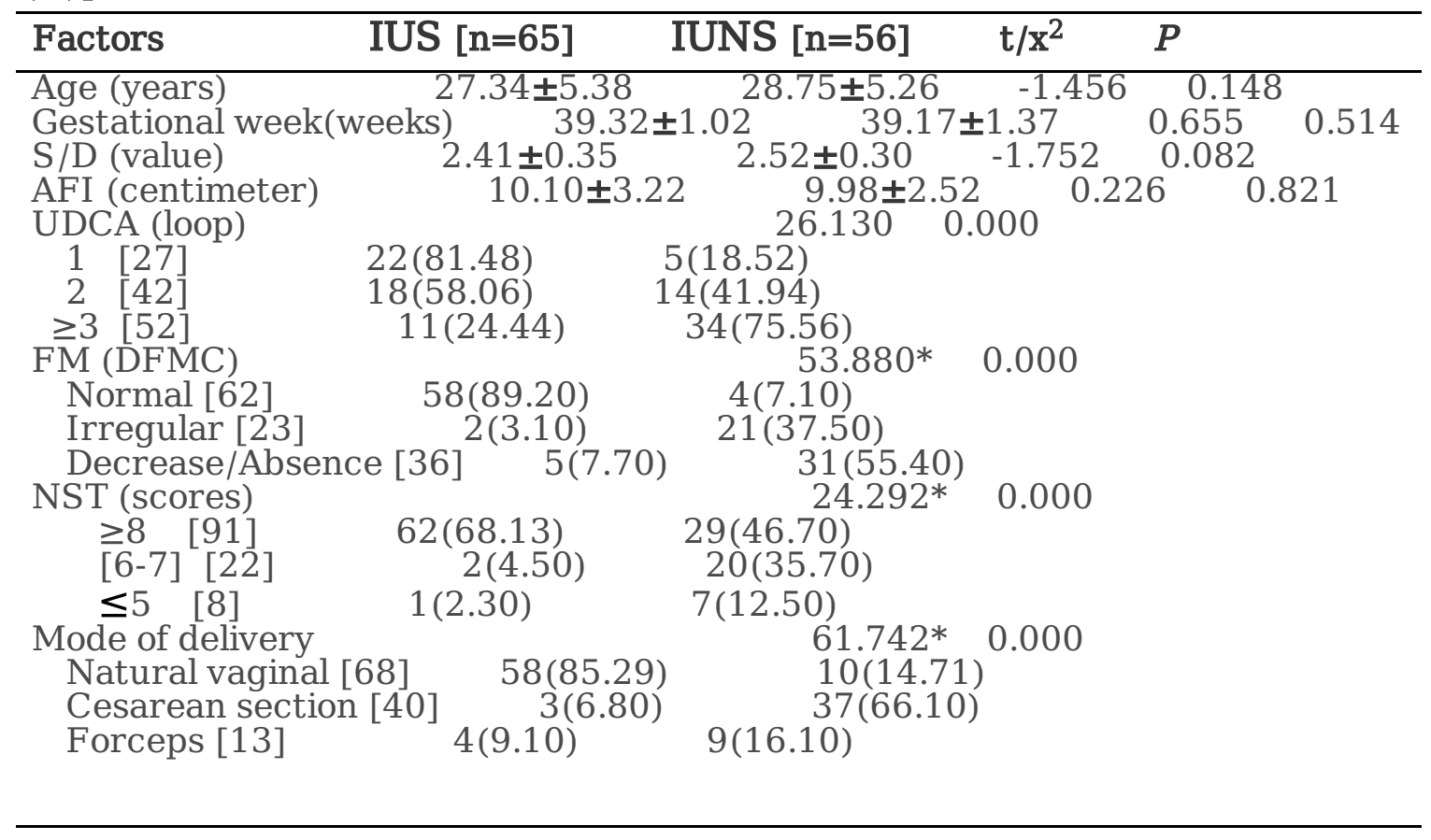

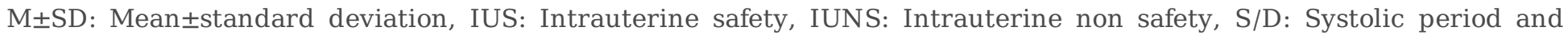
diastolic period blood flow ratio of umbilical artery under ultrasound. AFI: Amniotic fluid index. *showed by Chi-square test. 
The results of binary logistic stepwise regression analysis showed that DFMC and mode of delivery were the influencing factors of the outcome of fetal intrauterine security, $F M(W a l d=13.286, P=0.000)$ and mode of delivery (Wald $=21.816, P=0.000)$ in detail. The $\operatorname{Exp}(B)$ and $(95 \% \mathrm{Cl})$ of abnormal DFMC were separately 22.065 (4.155-117.178) for irregular FM and 21.282 (3.443-131.588) for decrease FM compared with normal FM (Table 2).

Table 2 Logistic regression analysis of high-risk factors that effects fetal intrauterine safety.

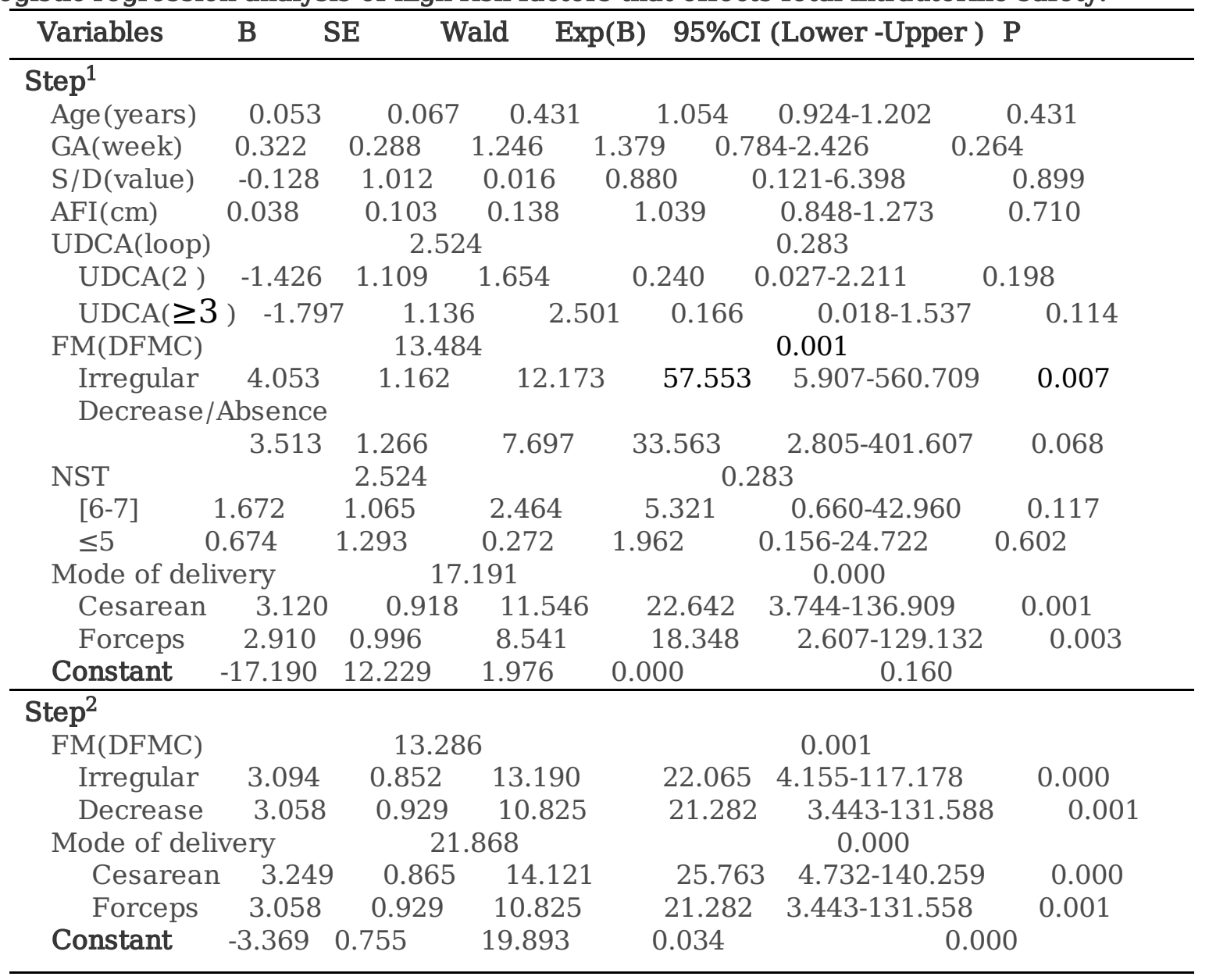

B: Partial regression index coefficient, SE: Standard Error, Wald: the square of B/SE. Exp (B): Odds Ration. Note: binary logistic stepwise regression analysis, Dependent variable: fetal intrauterine safety (safety: 0; non safety: 1), Independent variables: Age, gestational week, S/D, AFI, UDCA (1-2 loops: 0; $\geq 3$ loops: 1), DFMC (regular: 0; irregular: 1; Decrease/absence: 2), NST ( $\geq 8: 0 ; 6-7: 1 ; \leq 5: 2)$, Mode of delivery (natural vaginal: 0; Cesarean section: 1; Forceps: 2 ).

\section{Comparison of DFMC predictive evaluation before delivery and retrospective evaluation after delivery}

There was no significant difference between DFMC prediction before delivery and clinical retrospective evaluation of intrauterine fetal safety after delivery, all $\mathrm{P}$ value of different mode of delivery showed greater than 0.05 , only except for mode of cesarean delivery showed great significance (Table 3 ). This result completely confirmed the conclusion that the predictive value of DFMC was basically consistent with the clinical outcome in the mode of natural vaginal delivery. And the predictive efficacy of DFMC in different delivery modes showed natural vaginal delivery was the best, PPV, NPV, sensitivity, specificity 
and Yuden index were respectively $96.7 \%, 64.3 \%, 86.5 \%, 90.0 \%$ and 0.77 . Besides, the predictive efficacy of DFMC in cesarean delivery was the second, but with the highest specificity of $94.6 \%$ (Table 3 ).

Table 3 the comparisons between DFMC prediction and clinical results under different delivery modes and evaluating indicators of DFMC (\%).

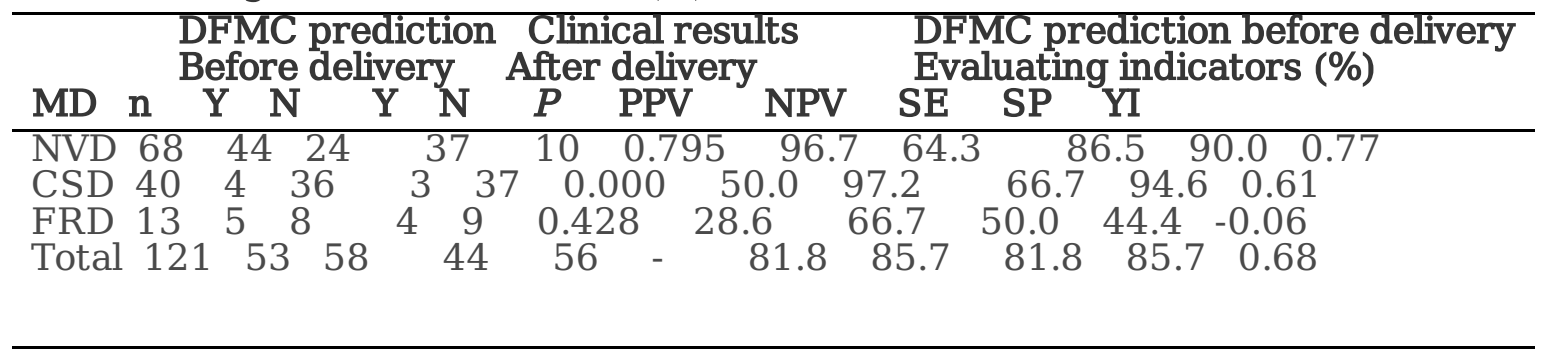

MD: Model of delivery, n: case number, Y: safety, N: no safety, PPV: Positive prediction value, NPV: Negative prediction value, SE: Sensibility, SP: Specificity, YI: Yuden's Index.

\section{Analysis of the distribution of reasons for cesarean section delivery}

The distribution of prenatal reasons for 40-case pregnant women by cesarean section delivery showed that the proportion of abnormal DFMC, fetal position and UCAN ( $\geq 3$ loops) were separately $87.5 \%, 62.5 \%$ and $62.5 \%$. This result also showed that the predictive value of DFMC on fetal intrauterine safety was the best. And the distribution of postpartum reasons showed the proportion of amniotic fluid ( $\geq$ II) was the highest, with the rate of $47.5 \%$, followed by asphyxia(Y) and Apgar's score $(\leq 6)$, with the rates of $40.0 \%$ and $35.0 \%$ (Table 4).

\section{Table 4 Distribution of reasons for 40-case cesarean section delivery [n (\%)]}



E-CS=Emergency CS, S-CS=Selective CS, P-ECS=Proportion of emergency CS, A-DFMC=Abnormal DFMC, FS=Fetal Stress, FP=Fetal Position, Y: asphyxia, N: non asphyxia. UCAN=Umbilical cord around neck.

\section{Discussion}

FM is a kind of activity mode in the process of fetal growth and development in the uterus. Clinical description of such as rolling, leg kicking, burp-like and other forms of FM, it is a good physiological reaction of fetus in the uterus $[2,4,5]$. From 18 to 20 gestational weeks, the pregnant woman can perceive or touch the FM. A normal FM should include FM count and regularity, and the former mainly describes FM count including excessive FM, decreased FM and absence of FM, the latter does the regularity of FM including normal peak-time FM and non peak-time FM [16]. Every fetal FM is not completely consistent, and pregnant women's activities, especially regularity of feeding is closely related [16]. The fetus will have abnormal FM when the intrauterine environment is adverse, including the increase, decrease and disappearance of FM $[2,3]$. In 1976, Pearson et al retrospectively analyzed the 
counting of fetal movements of pregnant women and found that FM could predict the intrauterine fetal safety. Some researchers all showed that the DFMC could successfully predict and evaluate the fetal safety in uterus by improving the modified FM chart by the "counting-to-ten" method [11, 12]. In this study, we selected the term pregnant women with UCAN, and found that DFMC can reliably predict the safety of the fetus with UCAN in the uterus before delivery. In comparison of the two groups, AFI was not significant, and the results were not consistent with those of previous studies $[17,18,19]$ which were positive correlation between AFI and fetal safety in uterus, and this reason may be related to the inconsistent criteria for inclusion of pregnant women without any clear prenatal risk factors. In this study, we found that the fetal intrauterine safety may be related to the loops of UCAN, DFMC, modified Fischer score (NST), S/D and mode of delivery by comparison of the prenatal factors. The loop of UCAN was found to be correlated with the safety of the fetus in the uterus, the more the loops of UCAN, the more lower the safety of the fetus in uterus, and other studies also had same $[20,21]$ or different results $[22$, 23]. FM is closely related to the number of UCAN, the length of umbilical cord, the degree of tightness and whether the knot is tied, which may indirectly affect the assessment of fetal safety in uterus [20, 24]. In order to further solve the possible bias of research results caused by the mutual influence or interference among risk factors in this study, the eight factors described above were included in the multi-variables logistic stepwise regression analysis, and it was clearly concluded that DFMC and mode of delivery were two independent risk factors affecting the intrauterine safety of fetus.

This study also showed that the mode of delivery, only except for mode of cesarean delivery, did not affect the effect of evaluation of fetal intrauterine safety. And DFMC had a better evaluation effect on the intrauterine safety of fetal with UCAN delivered by natural vaginal mode, while the sensitivity of evaluation of fetal safety in cesarean section mode is lower (66.7\%), but the specificity is higher (94.6\%), and the overall efficiency is low. And the proportion of cesarean section delivery in abnormal DFMC was the highest ( $87.5 \%$ ), followed by fetal position and UCAN ( $\geq 3$ loops), further confirmation of the predictive value of DFMC in prenatal prediction of fetal intrauterine safety $[22,25]$. And selective cesarean section delivery had improved the good outcome of perinatal infants with an increase of no asphyxia outcome from $47.5-60.0 \%$. We speculate that moderate expansion of cesarean section indications may reduce the adverse outcomes of perinatal infants, especially for those pregnant women with prenatal prediction of abnormal DFMC, UCAN for more than 3 loops, and amniotic fluid turbidity ( $\geq I I)$. And this result was in line with the suggestion of some obstetricians $[22,25,26]$.

Although the results of this study had been confirmed that DFMC can objectively and effectively predict and evaluate the intrauterine safety of fetus with UCAN, this study could still be very limited, firstly, research object of this study was too small and narrow, it could only enroll 100-case pregnant women and simple analyze normal pregnant women without high risk factors. Secondly, although we controlled for important known confounders, abdominal wall hypertrophy, education status and cognitive ability of FM also could confound the result of evaluation of FM [27]. And this confounded factor of cognitive ability of FM had been narrowed in this study by strictly selected study population. In other word, these 100-case pregnant women with UCAN all had been accepted this standardized training and testing how to perform this FM counting method called "count-to-ten" and how to record DFMC in the outpatient 
midwifery counseling agency, because this is the key to transform the subjective evaluation index of FM into an objective evaluation index of DFMC. So further studies should consider more problems described above. In view of many factors affecting FM, individualized FM monitoring may be the direction of further research in the future.

\section{Conclusions}

By use of this quantitative FM counting method and recording strategy, DFMC can objectively and effectively predict and evaluate the intrauterine safety of fetus with UCAN, and moderate expansion of the indications of cesarean section delivery for pregnant women with UCAN, which may reduce the adverse outcomes of perinatal infants.

\section{Abbreviations}

FM: fetal movement; DFMC: daily fetal movement counting chart; UCAN: umbilical cord around the neck; S/D: umbilical artery systolic blood flow to diastolic blood flow ratio; AFI: amniotic fluid index; NST: non stress test; NPV: negative predictive value; PPV: positive predictive value.

\section{Declarations}

\section{Acknowledgements:}

We would like to thank midwives from the outpatient of midwifery counseling agency in Zhejiang Quhua Hospital for their help with collection of date in this study.

\section{Authors' contributions}

ZJ Li: study design, overall administration and manuscript writing. X Zhou: study design, statistical analysis and language embellishment. YQ Zheng: statistical analysis and language embellishment. $\mathrm{M}$ Liu: partial administration, dates collection and partial training and testing of enrolled pregnancy women. XY Fu: partial administration and dates collection. AM Ye: overall training and testing of enrolled pregnancy women, and dates collection. All authors critically reviewed the manuscript and approved the final version.

\section{Funding}

None

\section{Availability of data and materials}

The datasets used and/or analyzed during the current study are available from the corresponding author on reasonable request. 


\section{Ethics approval and consent to participate}

This study was approved by the Ethics Committee of Zhe-Jiang Quhua Hospital (ECZJQH-202100018), all enrolled participants were well informed consent and voluntary accepted requirements for enrolled conditions. And all methods were performed in accordance with the relevant guidelines and regulations.

\section{Consent for publication}

Not applicable.

\section{Competing interests}

The authors declared that they have no competing interests.

\section{References}

1. Mangesi L, Hofmeyr GJ, Smith V, Smyth RM. Cochrane Collaboration: Fetal movement counting for assessment of fetal wellbeing. Cochrane Database Syst Rev. 2015; 15(15):CD004909.

2. Mangesi L, Hofmeyr GJ. Fetal movement counting for assessment of fetal wellbeing. Cochrane Database Syst Rev. 2007; 24(1):1-23.CD004909.

3. Saastad E, Tveit JV, Flenady V, Stray-Pedersen B, Fretts RC, Børdahl PE, Frøen JF. Implementation of uniform information on fetal movement in a Norwegian population reduced delayed reporting of decreased fetal movement and stillbirths in primiparous women-a clinical quality improvement. BMC Res Notes. 2010; 3(1) :2.

4. Raynes-Greenow CH, Gordon A, Li Q, Hyett JA. A cross-sectional study of maternal perception of fetal movements and antenatal advice in a general pregnant population, using a qualitative framework. BMC Pregnancy Childbirth. 2013; 13: 32.

5. Nomura RM, Ferreira MV, Latif IO, Francisco RP, Zugaib M. Agreement between maternal perception of fetal movements and visualization by ultrasound. Rev Bras Ginecol Obstet. 2013; 35(2):5559.Portuguese.

6. Mohr Sasson A, Tsur A, Kalter A, Weissmann Brenner A, Gindes L, Weisz B. Reduced fetal movement: factors affecting maternal perception. J Matern Fetal Neonatal Med. 2015; 1-4.

7. Tveit JV, Saastad E, Stray-Pedersen B, Børdahl PE, Flenady V, Fretts R, Frøen JF. Reduction of late stillbirth with the introduction of fetal movement information and guidelines - a clinical quality improvement. BMC Pregnancy Childbirth. 2009; 9(32):1-10.

8. Chauveau L, Raia-Barjat T, Noblot E, Fanget C, Gallot D, Chaleur C. Évaluation des connaissances des femmes enceintes sur les mouvements actifs fœtaux lors de la visite de terme [Maternal knowledge of fetal movements in late pregnancy]. J Gynecol Obstet Biol Reprod (Paris). 2016; 45(4):360-365. French. 
9. Stacey T, Thompson JM, Mitchell EA, Ekeroma A, Zuccollo J, McCowan LM. Maternal perception of fetal activity and late stillbirth risk: findings from the Auckland Stillbirth Study. Birth 2011, 38(4):311-316.

10. Notice of Zhejiang Health Department on Printing and Distributing the Administrative Measures for High-risk Pregnancy in Zhejiang Province (ZJSP23-2013-0006), issued by Zhejiang Health Department, 2013, No.182. website: http://wsjkw.zj.gov.cn

11. Winje BA, Saastad E, Gunnes N, Tveit JV, Stray-Pedersen B, Flenady V, Frøen JF. Analysis of 'count-toten' fetal movement charts: a prospective cohort study. BJOG. 2011;118(10):1229-1238.

12. Winje BA, Røislien J, Frøen JF. Temporal patterns in count-to-ten fetal movement charts and their associations with pregnancy characteristics: a prospective cohort study. BMC Pregnancy Childbirth. 2012; $12: 124$.

13. Hofmeyr GJ, Novikova N. Management of reported decreased fetal movements for improving pregnancy outcomes. Cochrane Database Syst Rev. 2012; 4(4):CD009148.

14. Delaram M, Jafarzadeh L. The Effects of Fetal Movement Counting on Pregnancy Outcomes. J Clin Diagn Res. 2016; 10(2):SC22-4.

15. Rådestad I, Lindgren H. Women's perceptions of fetal movements in full-term pregnancy. Sex Reprod Healthc. 2012; 3(3):113-116.

16. Kuwata T, Matsubara S, Ohkusa T, Ohkuchi A, Izumi A, Watanabe T, Suzuki M. Establishing a reference value for the frequency of fetal movements using modified 'count to 10' method. $J$ Obstet Gynaecol Res. 2008; 34(3):318-323.

17. Khooshideh M, Izadi S, Shahriari A, Mirteymouri M. The predictive value of ultrasound assessment of amniotic fluid index, biophysical profile score, non stress test and fetal movement chart for meconium-stained amniotic fluid in prolonged pregnancies. J Pak Med Assoc. 2009; 59(7):471-474.

18. Morris JM, Thompson K, Smithey J, Gaffney G, Cooke I, Chamberlain P, Hope P, Altman D, MacKenzie IZ. The usefulness of ultrasound assessment of amniotic fluid in predicting adverse outcome in prolonged pregnancy: a prospective blinded observational study. BJOG. 2003; 110(11):989-994.

19. Sherer DM, Ward K, Bennett M, Dalloul M. Current Perspectives of Prenatal Sonographic Diagnosis and Clinical Management Challenges of Nuchal Cord(s). Int J Womens Health. 2020; 10(12):613631.

20. Cho FN, Liu CB, Li JY, Carey JR, Liou WS. Absent fetal movement and brain sparing effect associated with multiple tight nuchal cords. Taiwan J Obstet Gynecol. 2013; 52(3):457-459.

21. Wang Y, Le Ray C, Audibert F, Wagner MS. Management of nuchal cord with multiple loops. Obstet Gynecol. 2008; 112 (2Pt2):460-461.

22. Kong CW, Lee DH, Chan LW, To WW. Impact of nuchal cord on fetal outcomes, mode of delivery, and management: a questionnaire survey of pregnant women. Hong Kong Med J. 2015; 21(2):143-148.

23. Sheiner E, Abramowicz JS, Levy A, Silberstein T, Mazor M, Hershkovitz R. Nuchal cord is not associated with adverse perinatal outcome. Arch Gynecol Obstet. 2006; 274: 81-83. 
24. Fangui Zhao, Qiuying Geng, Fanbin Kong1, Yan Ning. Quantitative analysis of tightness of nuchal cord and its relationship with fetal intrauterine distress. Int J Clin Exp Med. 2015; 8(10):1750717514.

25. Kong CW, Chan LW, To WW. Neonatal outcome and mode of delivery in the presence of nuchal cord loops: implications on patient counselling and the mode of delivery. Arch Gynecol Obstet. 2015; 292 (2): 283-289.

26. Kamalifard M, Abbasalizadeh S, Ghojazadeh M, Ghatreh Samani F, Rabiei L. Diagnostic value of fetal movement counting by mother and the optimal recording duration. J Caring Sci. 2013; 2(2):8995.

27. Saastad E, Winje BA, Israel P, Froen JF. Fetal movement counting-maternal concern and experiences: A multicentre, randomized, controlled trial. Birth. 2012; 39:10-20.

\section{Figures}

340 cases termed pregnant women with a gestational age between 37 and 41 weeks and UCAN confirmed by B-mode Ultrasound examination.

To exclude 105 cases with high-risk factors

235 cases pregnant women had accepted the standardized training and assessment of how to perform the DFMC.

To exclude 87 cases because of unaccomplished training, midway out or failure assessment .

148 cases pregnant women

To exclude 26 cases because of incomplete information

122 cases pregnant women

To kick out 22 cases because of non UCAN finally confirmed by review of delivery records.

100 cases pregnant women

Figure 1 
Research object screening flowchart

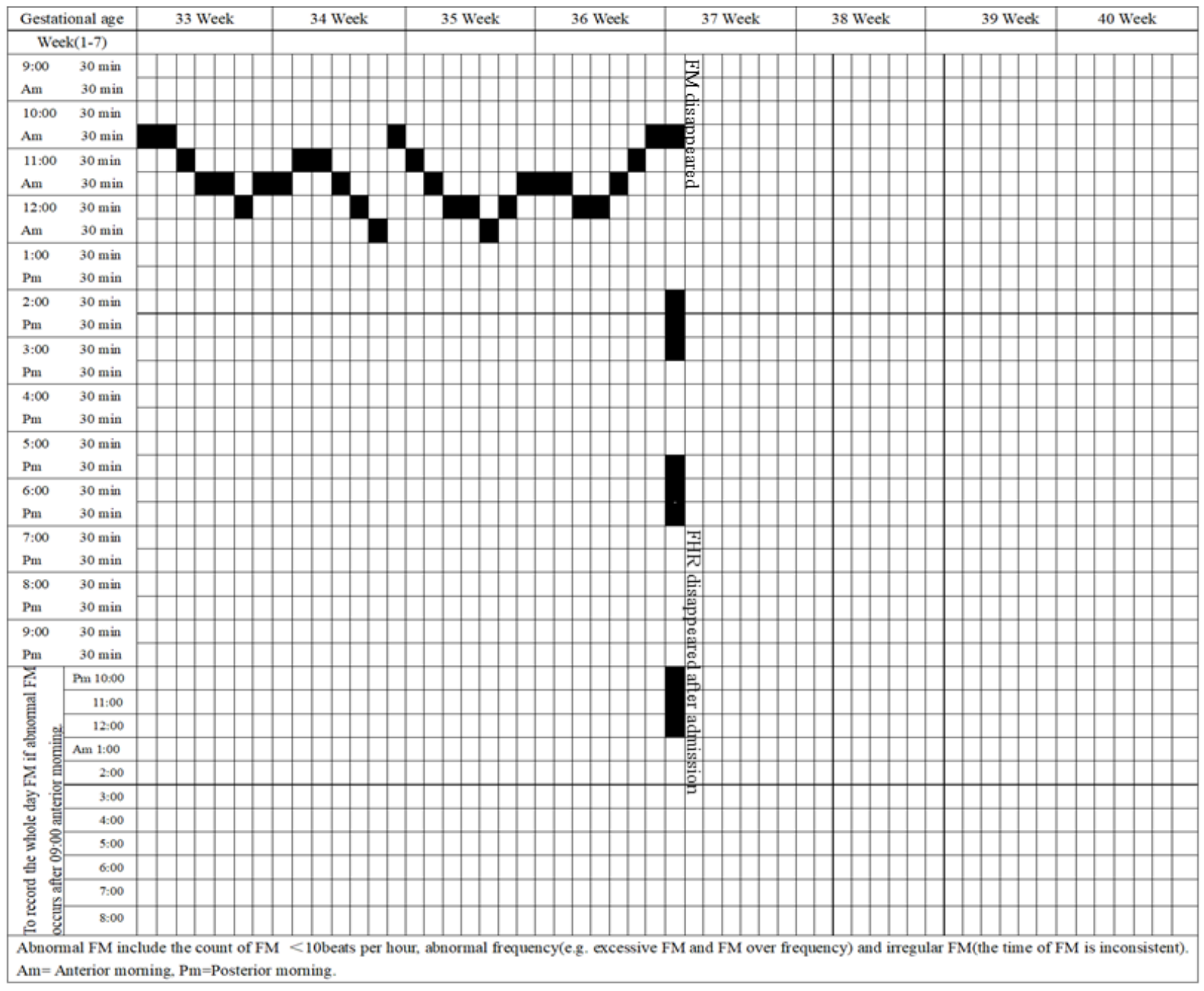

Figure 2

Daily fetal movement count chart (DFMC) of obstetric: To show a case of continuous change of FM from firstly regular and normal FM between 33 and 36 week to excessive FM and then to the final disappearance of FM at the gestation age of 37 week and 1st day. 